http://dx.doi.org/10.32929/2446-8355.2019v28n3p268-279

\title{
DIVERSIDADE GENÉTICA EM CULTIVARES E LINHAGENS DE FEIJÃO COMUM (Phaseolus vulgaris L.) UTILIZANDO ANÁLISES MULTIVARIADAS
}

\author{
Paulo Henrique Moron Pereira Leite ${ }^{1}$, Valvenarg Pereira da Silva ${ }^{2 *}$, Thiago Alexandre \\ Santana Gilio ${ }^{3}$, Rafhael Felipin-Azevedo ${ }^{4}$, Taniele Carvalho de Oliveira ${ }^{5}$, Marco Antonio \\ Aparecido Barelli ${ }^{6}$
}

\footnotetext{
${ }^{1}$ Engenheiro agrônomo - Universidade do Estado de Mato Grosso - Campus de Cáceres-Mato Grosso.

${ }^{2}$ Doutor em Biodiversidade e Biotecnologia, Professor do Curso de Ciências Biológicas da Universidade do Estado de Mato Grosso - Campus de Cáceres-MT. *E-mail do autor correspondente: silvabiologo@hotmail.com.

${ }^{3}$ Doutor em Melhoramento Genético de Plantas, Professor do Programa de Pós-Graduação em Genética e Melhoramento de Plantas - Universidade do Estado de Mato Grosso.

${ }^{4}$ Doutor em Melhoramento Genético de Plantas - Pós doutorando no programa de Genética e Melhoramento de Plantas.

${ }^{5}$ Engenheira agrônoma, Doutoranda do Programa de Pós-Graduação em Biodiversidade e Biotecnologia (Rede Bionorte) - Universidade do Estado de Mato Grosso.

${ }^{6}$ Doutor em Agronomia, Professor Adjunto do curso de Agronomia/ Programa de Pós-Graduação em Genética e Melhoramento de Plantas. Universidade do Estado de Mato Grosso - Campus de Cáceres-Mato Grosso.
}

Recebido: 30/11/2018; Aceito: 10/09/2019

RESUMO: O objetivo deste trabalho foi avaliar a divergência genética entre cultivares/ linhagens de feijão comum e indicar genitores contratantes e superiores para produzir populações segregantes com alta variabilidade. Avaliou-se 25 cultivares/linhagens de feijão, em delineamento de blocos ao acaso com três repetições. Os caracteres agronômicos avaliados foram: Número de dias para o florescimento, Ciclo, Altura média final das plantas, Altura média da inserção primeira vagem, Comprimento médio longitudinal das vagens, Número médio de vagens por planta, Número médio de sementes por vagem, Número médio de sementes por planta, Peso médio de 100 sementes e Produtividade de grãos. Os dados obtidos foram submetidos à análise de variância, seguido do teste de agrupamento de Scott e Knott. Para a análise da divergência genética entre as cultivares/linhagens foi empregada análise multivariada com base na distância generalizada de Mahalanobis, realizando posteriormente os métodos aglomerativos de otimização de Tocher, método hierárquico "UPGMA" e projeção de distância no plano 2D. O grau de preservação das distâncias genéticas no dendrograma foi verificado a partir do Coeficiente de Correlação Cofenética. Utilizou-se, também, o critério de Singh (1981) para quantificar a contribuição relativa das características para a divergência genética. As cultivares/linhagens avaliadas apresentam dissimilaridade genética quanto às características agronômicas avaliadas. A característica comprimento médio longitudinal das vagens é a que mais contribuiu para estimar a diversidade genética entre as cultivares/linhagens. E híbridos com maior efeito heterótico podem ser obtidos dos cruzamentos entre Iraí $\times$ Goiano Precoce, Iraí $\times$ Pérola, Goiano Precoce $\times$ CNFC 10467 e IAPAR $81 \times$ Pérola. 
Palavras-chave: Dissimilaridade. Melhoramento genético. Distância generalizada de Mahalanobis.

\title{
GENETIC DIVERSITY IN COMMON BEAN (Phaseolus vulgaris L.) CULTIVARS AND LINES USING MULTIVARIATE ANALYZES
}

\begin{abstract}
The objective of this work was to evaluate the genetic divergence between common bean cultivars/lines and indicate contrasting and superior parents to produce segregating populations with high variability. Twenty-five cultivars/lines of common bean were evaluated in a randomized complete block design with three replicates. The agronomic characters evaluated were: Number of days to flowering, Cycle, Final average height of plants, Average height of the first pod insert, Average longitudinal length of pods, Average number of pods per plants, Average number of seeds per pod, Average number of seeds per plant, Average weight of 100 seeds, Grain yield. Data were submitted to analysis of variance, followed by the Scott and Knott's clustering test. For the analysis of the genetic divergence among the cultivars/lines, a multivariate analysis based on the Mahalanobis generalized distance was used, performing later the agglomerative methods of tocher and hierarchical optimization "UPGMA" and distance in 2D projection plane. The degree of preservation of the genetic distances in the dendogram was verified from the Cophenetic Correlation Coefficient. The criterion of Singh (1981) was also used to quantify the relative contribution of the characteristics to the genetic divergence. The evaluated cultivars/lines show genetic dissimilarity regarding the agronomic traits evaluated. The average longitudinal length of pods was the trait that most contributed to estimate the genetic diversity among the cultivars/lines. Hybrids with greater heterotic effect can be obtained from the crossings between Iraí $\times$ Goiano Precoce, Iraí $\times$ Pérola, Goiano Precoce $\times$ CNFC 10467 and IAPAR 81 $\times$ Pérola.
\end{abstract}

Key words: Dissimilarity. Genetic plant breeding. Generalized distance of Mahalanobis.

\section{INTRODUÇÃO}

Os programas de melhoramento genético de feijão comum são baseados quase que exclusivamente na hibridação de cultivares e obtenção de linhagens superiores a partir de populações segregantes (TSUTSUMI et al., 2015). A escolha dos genitores para compor o bloco de hibridação é uma etapa crucial, devendo ser realizada de forma minuciosa e criteriosa, uma vez que dela depende o sucesso das etapas subsequentes do programa de melhoramento (VALE et al., 2015).

Dentre os métodos utilizados para escolha dos genitores, os modelos preditivos, com base na distância genética têm sido amplamente utilizados por diversos pesquisadores (GONÇALVES et al., 2016; SEBIM et al., 2016; MAZIERO et al., 2017; SULBACHER et al., 2017; VIDYAKAR et al., 2017). Este modelo dispensa a obtenção prévia das combinações híbridas, pois considera as diferenças agronômicas, morfológicas, fisiológicas 
e/ou moleculares expostas pelos genitores na determinação da variabilidade genética (CRUZ et al., 2014).

Esta variabilidade genética está sob constante processo de seleção nos ambientes onde são cultivados, e o cultivo intensivo com emprego de poucos cultivares que constitui uma exigência de mercado da agricultura moderna (TSUTSUMI et al., 2015) contribui para a redução da variabilidade genética. Bitocchi et al. (2013), aponta que cultivares de feijões de origem mesoamericana sofreram redução de $72 \%$ da diversidade genética durante o processo de domesticação.

Sabendo que cultivares registradas e linhagens tradicionais de feijão comum são utilizados em diversos programas de melhoramento de feijão comum, por possuírem características favoráveis, esta pesquisa teve como objetivo estimar a divergência genética entre cultivares e linhagens de feijão comum e indicar genitores contratantes e superiores para produzir populações segregantes com alta variabilidade.

\section{MATERIAL E MÉTODOS}

O experimento foi conduzido na unidade experimental da Empresa Mato-grossense de Pesquisa, Assistência e Extensão Rural (EMPAER), no município de Cáceres - MT, situada na latitude $16^{\circ} 43^{\prime} 42^{\prime} \mathrm{S}$ e longitude $57^{\circ} 40^{\prime} 51^{\prime \prime} \mathrm{W}$ cerca de $118 \mathrm{~m}$ de altitude. O clima da região, segundo a classificação de Köppen, é do tipo Awa, quente e úmido com inverno seco, com período de regime de chuvas variando de outubro a março, e de seca de abril a setembro (DALLACORT et al., 2014). O solo é classificado como Argissolo Vermelho Amarelo Eutrófico chernossólico, de textura média argilosa (ARANTES et al., 2012).

Foram avaliados 25 cultivares/linhagens de feijão comum, sendo 11 com características de ciclo precoce (Novo Jalo, Carioca Similar, Carioca Pitoco, Jalo Precoce, Goiano Precoce, Iraí, BRS Radiante, Carioca, Bambuí, CNF 6911 e CNFM 7119) e 14 do grupo carioca (Pérola, Magnífico, BRS Pontal, IAPAR 81, CNFC 10408, CNFC 10410, CNFC 10429, CNFC 10431, CNFC 10432, CNFC 10438, CNFC 10444, CNFC 10455, CNFC 10467 e CNFC 10470 cedidas pela Embrapa Arroz e Feijão.

O delineamento experimental utilizado foi o de blocos ao acaso, com 25 tratamentos em três repetições em que cada parcela foi composta de quatro fileiras de plantas de quatro metros de comprimento, com espaçamento de $0,50 \mathrm{~m}$ e área útil de duas linhas centrais, com o plantio realizado no período de junho a setembro de 2005. Os tratos culturais foram realizados conforme recomendados para a cultura com irrigações periódicas em todas as fases de desenvolvimento das plantas.

Os caracteres agronômicos avaliados foram: Número de dias para o florescimento (FLORESC), Ciclo (CICLO), Altura média final das plantas (ALTP), Altura média da inserção primeira vagem (ALTINS), Comprimento médio longitudinal das vagens (CLMV), Número médio de vagens por planta (NMVP), Número médio de sementes por vagem (NMSV), Número médio de sementes por planta (NMSP), Peso médio de 100 sementes (PMS) e Produtividade de grãos (PG). 
Os dados obtidos foram submetidos à análise de variância, seguido do teste de agrupamento de médias Scott e Knott a $1 \%$ de probabilidade. Para a análise da divergência genética entre as cultivares/linhagens foi empregada análise multivariada com base na distância generalizada de Mahalanobis, realizando posteriormente os métodos aglomerativos de otimização de Tocher, hierárquico "UPGMA" e projeção de distância no plano 2D. O grau de preservação das distâncias genéticas no dendrograma foi verificado a partir do Coeficiente de Correlação Cofenética (CCC). Utilizou-se, também, o critério de Singh (1981) para quantificar a contribuição relativa das características para a divergência genética. Todas as análises foram realizadas utilizando os recursos computacionais do software Genes (CRUZ, 2013).

\section{RESULTADOS E DISCUSSÃO}

A diversidade genética é pré-requisito determinante para obtenção de ganhos genéticos em programas de melhoramento genético vegetal (CRUZ et al., 2014). Como pode ser observado na tabela 1, os genótipos avaliados apresentaram diferenças estatísticas ao nível de $1 \%$ para as características FLORESC, CICLO, ALTP, ALTINS, CLMV, NMVP, NMSV, NMSP, PMS, permitindo assim inferir sobre a existências de variabilidade genética nos genótipos analisados na presente pesquisa. Apenas a características PG não apresentou diferença estatísticas enfatizando a necessidade de desenvolvimento de novas cultivares.

Tabela 1. Resumo da análise de variância, com base na média de dez características agronômicas avaliadas dos 25 cultivares/linhagens de feijão comum, avaliadas no município de Cáceres-Mato Grosso. Summary of the analysis of variance, based on the average of ten agronomic characteristics evaluated from the 25 common bean cultivars, evaluated in the city of Cáceres, state of Mato Grosso.

\begin{tabular}{|c|c|c|c|c|c|c|}
\hline \multicolumn{7}{|c|}{ Quadrados Médios } \\
\hline FV & GL & FLORESC & CICLO & ALTP & ALTINS & CLMV \\
\hline Blocos & 2 & 0,89 & 0,64 & 189,00 & 5,79 & 0,66 \\
\hline Cultivares & 24 & $23,08 * *$ & $13,00 * *$ & $166,37 * *$ & $13,29 * *$ & $4,30 * *$ \\
\hline Resíduo & 48 & 0,56 & 0,84 & 16,55 & 4,80 & 0,20 \\
\hline Média & & 44,06 & 75,19 & 53,42 & 14,98 & 10,80 \\
\hline $\mathrm{CV}(\%)$ & & 1,69 & 1,22 & 7,62 & 14,64 & 4,22 \\
\hline \multicolumn{7}{|c|}{ Quadrados Médios } \\
\hline $\mathrm{FV}$ & GL & NMVP & NMSP & NMSV & PMS & $\mathrm{PG}$ \\
\hline Blocos & 2 & 3,53 & 600,44 & 1,57 & 17,50 & 151,18 \\
\hline Cultivares & 24 & $66,57 * *$ & $3276,16 * *$ & $0,74 * *$ & $64,73 * *$ & $2104,95^{\mathrm{ns}}$ \\
\hline Resíduo & 48 & 22,98 & 980,54 & 0,24 & 3,55 & 1108,14 \\
\hline Média & & 26,53 & 149,60 & 5,60 & 24,46 & 136,30 \\
\hline $\mathrm{CV}(\%)$ & & 18,07 & 20,93 & 8,78 & 7,70 & 24,42 \\
\hline
\end{tabular}

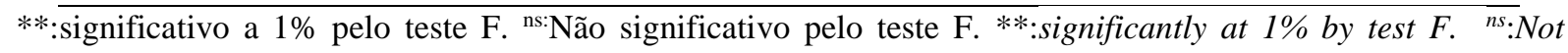
significantly of test $F$.

Além da divergência genética os programas de melhoramento devem levar em consideração o desempenho produtivo dos genótipos, analisando criteriosamente 
características ligadas a precocidade e produtividade. Sendo assim, a cultivar Iraí se mostrou como a mais precoce, com florescimento e ciclo médio de 38,66 e 70 dias, respectivamente (Tabela 2). A performance desta cultivar no quesito precocidade, está acima do desempenho da linhagem da CNFP 10794 observada por Ribeiro et al. (2018) como a mais precoce, com ciclo de 78,20 dias e com desempenho muito próximo da cultivar superprecoce BRS FC104 de ciclo de 65 dias (MELO et al., 2017).

Tabela 2. Análise de agrupamento de médias Scott e Knott das 25 cultivares/linhagens de feijão comum em relação às dez características avaliadas, no município de Cáceres-Mato Grosso. Cluster analysis of Scott and Knott averages of the 25 common bean cultivars in relation to the ten characteristics evaluated, in the city of Cáceres state of Mato Grosso.

\begin{tabular}{cccccc}
\hline & \multicolumn{5}{c}{ Médias $^{1 /}$ das características ${ }^{\prime}{ }^{\prime}$ avaliadas } \\
\hline Cultivares & FLORESC & CICLO & ALTP & ALTINS & CLMV \\
\hline Novo Jalo & $41,33 \mathrm{~d}$ & $72,33 \mathrm{c}$ & $43,26 \mathrm{c}$ & $11,96 \mathrm{~b}$ & $13,75 \mathrm{a}$ \\
Carioca Similar & $42,33 \mathrm{c}$ & $76,00 \mathrm{a}$ & $56,80 \mathrm{~b}$ & $14,86 \mathrm{~b}$ & $10,31 \mathrm{e}$ \\
Carioca Pitoco & $44,33 \mathrm{~b}$ & $75,33 \mathrm{~b}$ & $53,06 \mathrm{~b}$ & $17,43 \mathrm{a}$ & $10,45 \mathrm{e}$ \\
Jalo Precoce & $41,00 \mathrm{~d}$ & $71,66 \mathrm{c}$ & $43,80 \mathrm{c}$ & $13,40 \mathrm{~b}$ & $12,73 \mathrm{~b}$ \\
Goiano Precoce & $38,66 \mathrm{e}$ & $72,00 \mathrm{c}$ & $36,26 \mathrm{~d}$ & $14,63 \mathrm{~b}$ & $10,30 \mathrm{e}$ \\
Iraí & $38,66 \mathrm{e}$ & $70,00 \mathrm{~d}$ & $46,40 \mathrm{c}$ & $17,20 \mathrm{a}$ & $13,05 \mathrm{~b}$ \\
BRS Radiante & $38,66 \mathrm{e}$ & $71,33 \mathrm{c}$ & $52,36 \mathrm{~b}$ & $19,30 \mathrm{a}$ & $12,46 \mathrm{c}$ \\
Carioca & $44,33 \mathrm{~b}$ & $76,00 \mathrm{a}$ & $52,13 \mathrm{~b}$ & $15,73 \mathrm{a}$ & $10,43 \mathrm{e}$ \\
Bambuí & $44,00 \mathrm{~b}$ & $74,66 \mathrm{~b}$ & $46,80 \mathrm{c}$ & $14,63 \mathrm{~b}$ & $10,41 \mathrm{e}$ \\
CNFC 6911 & $44,00 \mathrm{~b}$ & $74,33 \mathrm{~b}$ & $48,33 \mathrm{c}$ & $16,06 \mathrm{a}$ & $10,28 \mathrm{e}$ \\
CNFM 7119 & $40,33 \mathrm{~d}$ & $74,66 \mathrm{~b}$ & $43,00 \mathrm{c}$ & $18,66 \mathrm{a}$ & $9,86 \mathrm{f}$ \\
Pérola & $46,00 \mathrm{a}$ & $76,66 \mathrm{a}$ & $62,25 \mathrm{a}$ & $14,26 \mathrm{~b}$ & $11,91 \mathrm{c}$ \\
Magnífico & $47,66 \mathrm{a}$ & $77,33 \mathrm{a}$ & $61,06 \mathrm{a}$ & $16,00 \mathrm{a}$ & $10,41 \mathrm{e}$ \\
BRS Pontal & $46,66 \mathrm{a}$ & $76,66 \mathrm{a}$ & $53,80 \mathrm{~b}$ & $13,20 \mathrm{~b}$ & $10,86 \mathrm{~d}$ \\
Iapar 81 & $46,66 \mathrm{a}$ & $76,00 \mathrm{a}$ & $66,06 \mathrm{a}$ & $16,73 \mathrm{a}$ & $11,18 \mathrm{~d}$ \\
CNFC 10408 & $44,00 \mathrm{~b}$ & $77,33 \mathrm{a}$ & $49,26 \mathrm{c}$ & $16,33 \mathrm{a}$ & $9,86 \mathrm{f}$ \\
CNFC 10410 & $44,66 \mathrm{~b}$ & $76,00 \mathrm{a}$ & $51,46 \mathrm{~b}$ & $11,46 \mathrm{~b}$ & $9,80 \mathrm{f}$ \\
CNFC 10429 & $45,33 \mathrm{a}$ & $76,66 \mathrm{a}$ & $63,26 \mathrm{a}$ & $14,80 \mathrm{~b}$ & $10,30 \mathrm{e}$ \\
CNFC 10431 & $45,66 \mathrm{a}$ & $76,00 \mathrm{a}$ & $61,00 \mathrm{a}$ & $11,93 \mathrm{~b}$ & $9,40 \mathrm{f}$ \\
CNFC 10432 & $46,00 \mathrm{a}$ & $77,00 \mathrm{a}$ & $59,20 \mathrm{a}$ & $13,23 \mathrm{~b}$ & $9,63 \mathrm{f}$ \\
CNFC 10438 & $46,33 \mathrm{a}$ & $76,66 \mathrm{a}$ & $57,93 \mathrm{a}$ & $16,46 \mathrm{a}$ & $10,16 \mathrm{e}$ \\
CNFC 10444 & $46,66 \mathrm{a}$ & $76,00 \mathrm{a}$ & $53,40 \mathrm{~b}$ & $12,56 \mathrm{~b}$ & $9,25 \mathrm{f}$ \\
CNFC 10455 & $46,33 \mathrm{a}$ & $76,66 \mathrm{a}$ & $59,80 \mathrm{a}$ & $12,46 \mathrm{~b}$ & $9,86 \mathrm{f}$ \\
CNFC 10467 & $46,33 \mathrm{a}$ & $76,66 \mathrm{a}$ & $58,13 \mathrm{a}$ & $15,26 \mathrm{a}$ & $11,30 \mathrm{~d}$ \\
CNFC 10470 & $45,66 \mathrm{a}$ & $76,00 \mathrm{a}$ & $56,66 \mathrm{~b}$ & $15,86 \mathrm{a}$ & $11,90 \mathrm{c}$ \\
\hline & & & &
\end{tabular}


Tabela 2. Continuação...

\begin{tabular}{cccccc}
\hline & & Médias $^{1 /}$ das características ${ }^{2}$ avaliadas & \\
\hline Cultivares & NMVP & NMSP & NMSV & PMS & PG $^{\mathrm{ns}}$ \\
\hline Novo Jalo & $21,13 \mathrm{~b}$ & $111,38 \mathrm{~b}$ & $5,26 \mathrm{~b}$ & $32,75 \mathrm{a}$ & 123,80 \\
Carioca Similar & $24,73 \mathrm{~b}$ & $144,31 \mathrm{~b}$ & $5,83 \mathrm{a}$ & $23,96 \mathrm{~b}$ & 134,75 \\
Carioca Pitoco & $20,86 \mathrm{~b}$ & $129,60 \mathrm{~b}$ & $6,06 \mathrm{a}$ & $22,83 \mathrm{~b}$ & 118,15 \\
Jalo Precoce & $15,40 \mathrm{~b}$ & $70,75 \mathrm{~b}$ & $4,63 \mathrm{~b}$ & $32,45 \mathrm{a}$ & 84,50 \\
Goiano Precoce & $19,93 \mathrm{~b}$ & $93,68 \mathrm{~b}$ & $4,70 \mathrm{~b}$ & $30,93 \mathrm{a}$ & 92,56 \\
Iraí & $28,26 \mathrm{a}$ & $146,08 \mathrm{~b}$ & $5,16 \mathrm{~b}$ & $35,36 \mathrm{a}$ & 200,08 \\
BRS Radiante & $23,80 \mathrm{~b}$ & $115,85 \mathrm{~b}$ & $4,86 \mathrm{~b}$ & $33,51 \mathrm{a}$ & 138,53 \\
Carioca & $27,53 \mathrm{a}$ & $170,46 \mathrm{a}$ & $6,16 \mathrm{a}$ & $22,81 \mathrm{~b}$ & 126,66 \\
Bambuí & $25,73 \mathrm{a}$ & $159,54 \mathrm{a}$ & $6,20 \mathrm{a}$ & $22,75 \mathrm{~b}$ & 132,26 \\
CNFC 6911 & $27,26 \mathrm{a}$ & $132,86 \mathrm{~b}$ & $4,93 \mathrm{~b}$ & $24,06 \mathrm{~b}$ & 110,80 \\
CNFM 7119 & $21,53 \mathrm{~b}$ & $117,59 \mathrm{~b}$ & $5,43 \mathrm{~b}$ & $26,00 \mathrm{~b}$ & 112,70 \\
Pérola & $27,95 \mathrm{a}$ & $159,10 \mathrm{a}$ & $5,66 \mathrm{a}$ & $23,13 \mathrm{~b}$ & 135,13 \\
Magnífico & $22,33 \mathrm{~b}$ & $123,76 \mathrm{~b}$ & $5,60 \mathrm{a}$ & $21,51 \mathrm{~b}$ & 98,55 \\
BRS Pontal & $27,93 \mathrm{a}$ & $177,42 \mathrm{a}$ & $6,40 \mathrm{a}$ & $22,25 \mathrm{~b}$ & 152,16 \\
Iapar 81 & $33,20 \mathrm{a}$ & $195,05 \mathrm{a}$ & $5,86 \mathrm{a}$ & $22,18 \mathrm{~b}$ & 159,78 \\
CNFC 10408 & $28,26 \mathrm{a}$ & $163,84 \mathrm{a}$ & $5,76 \mathrm{a}$ & $22,00 \mathrm{~b}$ & 152,18 \\
CNFC 10410 & $32,20 \mathrm{a}$ & $176,55 \mathrm{a}$ & $5,46 \mathrm{~b}$ & $22,13 \mathrm{~b}$ & 161,20 \\
CNFC 10429 & $22,33 \mathrm{~b}$ & $125,77 \mathrm{~b}$ & $5,63 \mathrm{a}$ & $20,93 \mathrm{~b}$ & 116,51 \\
CNFC 10431 & $29,20 \mathrm{a}$ & $159,10 \mathrm{a}$ & $5,46 \mathrm{~b}$ & $20,55 \mathrm{~b}$ & 123,85 \\
CNFC 10432 & $31,60 \mathrm{a}$ & $189,04 \mathrm{a}$ & $6,00 \mathrm{a}$ & $20,08 \mathrm{~b}$ & 158,13 \\
CNFC 10438 & $29,20 \mathrm{a}$ & $189,04 \mathrm{a}$ & $6,50 \mathrm{a}$ & $20,11 \mathrm{~b}$ & 159,93 \\
CNFC 10444 & $31,93 \mathrm{a}$ & $169,91 \mathrm{a}$ & $5,26 \mathrm{~b}$ & $22,73 \mathrm{~b}$ & 140,38 \\
CNFC 10455 & $31,00 \mathrm{a}$ & $177,05 \mathrm{a}$ & $5,73 \mathrm{a}$ & $21,43 \mathrm{~b}$ & 152,00 \\
CNFC 10467 & $33,93 \mathrm{a}$ & $199,40 \mathrm{a}$ & $5,90 \mathrm{a}$ & $19,86 \mathrm{~b}$ & 170,26 \\
CNFC 10470 & $25,93 \mathrm{a}$ & $142,96 \mathrm{~b}$ & $5,53 \mathrm{~b}$ & $25,13 \mathrm{~b}$ & 152,60 \\
\hline
\end{tabular}

${ }^{1 /}$ Médias seguidas de mesma letra não diferem significativamente a $1 \%$ de probabilidade pelo teste Scott e Knott. ${ }^{2 /}$ FLORESC = Número de dias para o florescimento; CICLO = Ciclo; ALTP = Altura média final das plantas; ALTINS = Altura média da inserção primeira vagem; CLMV = Comprimento médio longitudinal das vagens; NMVP = Número médio de vagens por planta; NMSV = Número médio de sementes por vagem; NMSP = Número médio de sementes por planta; PMS = Peso médio de 100 sementes; PG = Produtividade de grãos. ${ }^{1 / M e a n}$ followed by the same letter do not differ significantly at of $1 \%$ probability by the Scott Knott test. ${ }^{2}$ FLOWER = Number of days for flowering; CYCLE = Cycle; AHP = Average Height of plants; HFPI = Height of first pod insertion; ALLP = Average longitudinal length of the pods; ANPP = Average number of pods per plant; ANSP = Average number of seeds per pods; ANSPL = Average number of seed per plant; AWS = Average weight of 100 seeds; GP = Grain productivity.

Em relação a característica altura de plantas, busca-se cultivares que apresentam maior altura, uma vez que esta característica apresenta relação com rendimento de grãos. Com isso as cultivares PÉROLA e FTS MAGNÍFICO e as linhagens CNFC 10429, CNFC 10431, CNFC 10432, CNFC 10438, CNFC 10455 e CNFC 10467 se sobressaíram apresentando altura de 58,13 a 63,26 cm (Tabela 2), sendo as indicadas para programa de melhoramento que visem incremento para esta característica. 
Para altura da inserção da primeira vagem, as cultivares e linhagens avaliadas foram agrupadas em dois grupos pelo teste de Scott e Knott, em que o grupo com os melhores resultados apresentaram valores de 15,26 a 19,30 cm, e o grupo com menor valor de 11,46 a 11,86. Sendo assim, ambos os grupos apresentam valores aceitáveis para esta característica, uma vez que as estimativas devem ser superiores à $12 \mathrm{~cm}$, para facilitar a colheita mecanizada e diminuir de perdas causadas por doenças fúngicas ocasionadas pelo contato das vagens à umidade do solo (OLIVEIRA et al., 2014; GONÇALVES et al., 2016).

O comprimento médio longitudinal das vagens variou 13,75 a $9,25 \mathrm{~cm}$, dividindo as cultivares / linhagens em seis grupos, em que a cultivar Novo Jalo apresentou o melhor resultado de 13,75 cm (Tabela 2). Este resultado é superior ao da pesquisa realizada por Poletine et al. (2016), que avaliou 40 genótipos de feijão na região de Arenito Caiuá no noroeste do estado do Paraná em que o melhor resultado foi de $11,90 \mathrm{~cm}$.

Para número médio de vagens por plantas as cultivares e linhagens ficaram divididas em dois grupos, o primeiro com valores de 15,50 a 24,73 e o segundo com melhor resultado de 27,73 a 33,93 vagens por plantas (Tabela 2). O resultado do melhor grupo é superior aos resultados obtidos por Mambrin et al. (2015) avaliando 14 linhagens avançadas de feijão e por Gonçalves et al. (2016) avaliando 40 acessos tradicionais de feijão.

Com relação ao número médio de sementes por plantas e sementes por vagens as cultivares e linhagens foram divididas em dois grupos, as seguintes cultivares: Carioca, Bambuí, Pérola, BRS Pontal, Iapar 81 e as linhagens CNFC 10408, CNFC 10432, CNFC 10438, CNFC 10455 e CNFC 10467 obtiveram os melhores resultados para ambas as características (Tabela 1). Dentre os materiais citados acima alguns já se sobressaíram em outras pesquisas tais como a linhagem CNFC 10467 para número de sementes por vagens em Salgado et al. (2011) e a cultivar carioca para número de sementes por plantas e número de sementes por vagens em pesquisa realizada por Mambrin et al. (2015).

Para peso médio de 100 sementes, a média entre as cultivares e linhagens avaliados foi de 24,46 g, dividindo em dois grupos, com destaque para as cultivares Írai, BRS Radiante, Novo Jalo, Jalo Precoce e Goiano Precoce que apresentaram média superior de $30 \mathrm{~g}$ (Tabela 2). Estes resultados é um indicativo que estas cultivares pertencerem ao grupo gênico andino, uma vez que, como apontado por Gepts e Bliss (1986) e Coelho et al. (2007) peso de 100 sementes inferior a $25 \mathrm{~g}$ indicam feijão pertencente ao centro mesoamericano.

Em relação à importância relativa dos caracteres, a característica com maior contribuição relativa para a avaliação da diversidade genética entre as cultivares/linhagens no presente trabalho foi, segundo o método de Singh (1981), comprimento longitudinal médio das vagens (Tabela 3). Esta mesma característica foi a principal para discriminação da divergência genética em pesquisa de Grigolo et al. (2018), avaliando 20 acessos de feijão comum. Este fato sugere que está característica deve ser priorizada nos estudos de divergência genética do feijão comum.

A característica número médio de sementes por plantas, não contribuiu para a detecção da variabilidade genética na presente pesquisa, sendo que caracteres que contribuíram com um percentual muito baixo ou não contribuíram para a variabilidade detectada podem ser 
descartadas. Todavia, pelo fato de ser uma característica importante no processo produtivo, a mesma não deve ser descartada (CORREA; GONÇALVES, 2012).

Tabela 3. Contribuição relativa dos dez caracteres agronômicos para a divergência genética entre 25 cultivares de feijão comum do grupo carioca, Cáceres-Mato Grosso. Relative contribution of the ten agronomic characters to the genetic divergence among 25 common bean cultivars of the carioca group, city of Cáceres, state of Mato Grosso.

\begin{tabular}{cc}
\hline Características $^{1 /}$ & Importância relativa (\%) $^{\text {CLMV }}$ \\
FLORESC & 35,347 \\
NMSV & 18,307 \\
NMVP & 12,666 \\
ALTP & 11,095 \\
CICLO & 7,717 \\
PMS & 6,900 \\
PG & 5,786 \\
ALTINS & 1,143 \\
NMSP & 1,035 \\
\hline
\end{tabular}

$\overline{1 / F L O R E S C ~}=$ Número de dias para o florescimento; CICLO = Ciclo; ALTP = Altura média final das plantas; ALTINS = Altura média da inserção primeira vagem; CLMV = Comprimento médio longitudinal das vagens; NMVP = Número médio de vagens por planta; NMSV = Número médio de sementes por vagem; NMSP = Número médio de sementes por planta; PMS = Peso médio de 100 sementes; $\mathrm{PG}=$ Produtividade de grãos . ${ }^{2 /} F L O W E R=$ Number of days for flowering; CYCLE = Cycle; $A H P=$ Average Height of plants; HFPI = Height of first pod insertion; $A L L P=$ Average longitudinal length of the pods; ANPP $=$ Average number of pods per plant; ANSP = Average number of seeds per pods; ANSPL = Average number of seed per plant AWS $=$ Average weight of 100 seeds; GP = Grain productivity.

Tabela 4. Grupo de genótipos de feijão com padrões similares, estabelecido pelo método de Tocher, utilizando a Distância Generalizada de Mahalanobis como medida de dissimilaridade avaliados no município de Cáceres-Mato Grosso. Group of bean genotypes with similar patterns, established by the Tocher's method, using the generalized distance of Mahalanobis as a measure of dissimilarity evaluated in the city of Cáceres state of Mato Grosso.

\begin{tabular}{cc}
\hline Grupos & Cultivares/linhagens \\
\hline & Carioca Similar; Carioca Pitoco; Carioca ; Bambuí ; CNFC 6911; \\
I & Magnífico; BRS Pontal; Iapar 81; CNFC 10408; CNFC 10410; CNFC \\
& 10429; CNFC 10431; CNFC 10432; CNFC 10438; CNFC 10444; CNFC \\
& 10455; CNFC 10467 \\
\hline II & Pérola; CNFC 10470 \\
\hline III & Novo Jalo; Jalo Precoce; BRS Radiante; Iraí \\
\hline IV & Goiano Precoce; CNFM 7119 \\
\hline
\end{tabular}

Utilizando a distância generalizada de Mahalanobis, os 25 cultivares/linhagens foram agrupadas de forma semelhantes em quatro grupos, pelo método de otimização de Tocher (Tabela 4), pelo método UPGMA (Figura 1) e pela projeção 2D (Figura 2). Essa equivalência no agrupamento das cultivares/linhagens pelos métodos é uma evidência da consistência nos resultados, e o uso do dendrograma e da projeção 2D para visualização e dispersão da variabilidade genética são factíveis devido ao Coeficiente de Correlação Cofenética 
apresentar-se significativo para UPGMA $(0,90 * *)$ pelo teste t e a Projeção $2 \mathrm{D}$ apresentou uma alta correlação entre a matriz e a dispersão $(0,99)$, com baixa distorção $(7,87 \%)$ e estresse $(9,27 \%)$.

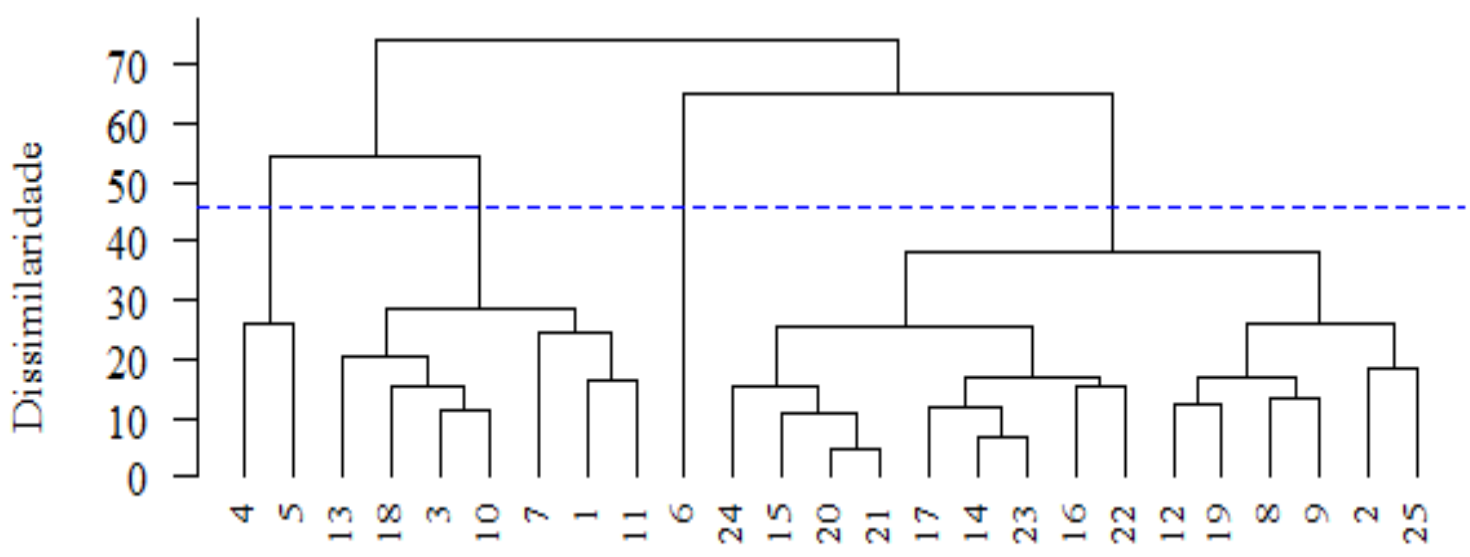

Figura 1. Dendrograma da divergência genética entre 25 cultivares/linhagens de feijão comum, obtido pelo método de ligações médias (UPGMA), utilizando a distância generalizada de Mahalanobis como medida de dissimilaridade. Dendogram of the genetic divergence among 25 cultivars of common bean, obtained by the average binding method (UPGMA), using the generalized distance of Mahalanobis as a measure of dissimilarity.

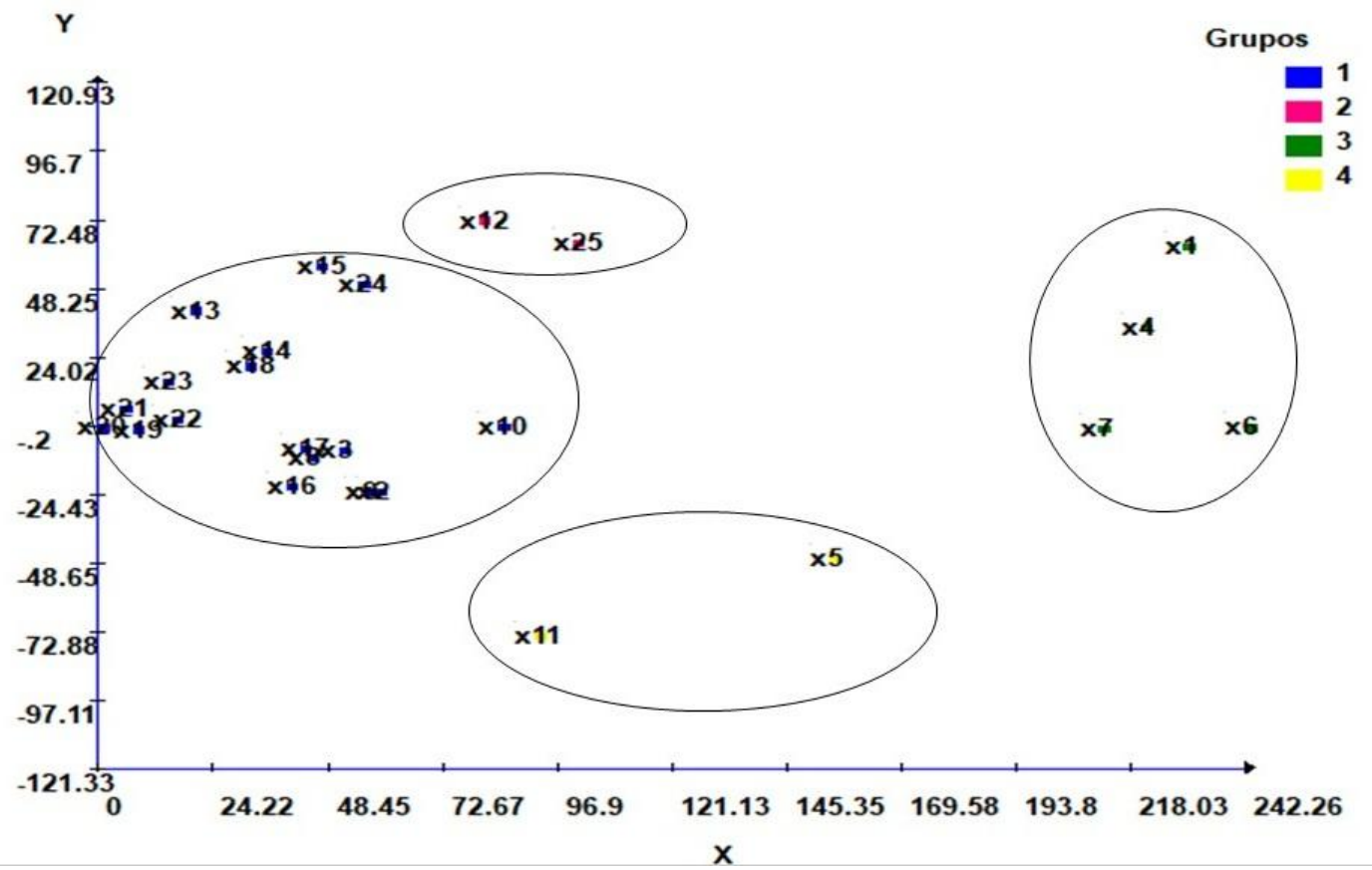

Figura 2. Projeção 2D, obtidas de dez características avaliadas em 25 cultivares/linhagens, Cáceres-MT. 2D projection, obtained from ten traits evaluated in 25 cultivars, city of Cáceres state of Mato Grosso.

A formação de grupos distintos confirma a existência de divergência genética, o que vem a ser um aspecto positivo do ponto de vista da utilização destes genótipos em hibridações artificiais com a finalidade de ampliar a variabilidade genética. Neste sentido, é indicado a escolha de genitores de grupos distintos por estes apresentarem maiores distâncias genéticas 
(ROTILI et al., 2012; VIDYAKAR et al., 2017). Contudo cabe ressaltar que para a escolha de genitores além de a contrastância genética é necessário a presença de características agronômicas desejáveis de acordo com o programa de melhoramento.

Desta maneira tomando como base os resultados dos agrupamentos e das características agronômicas avaliadas, recomenda-se os cruzamentos: Iraí $\times$ Goiano Precoce, Iraí $\times$ Pérola, Goiano Precoce $\times$ CNFC 10467 e IAPAR $81 \times$ Pérola para o desenvolvimento de populações segregantes com alta variabilidade genética.

\section{CONCLUSÃO}

As cultivares/linhagens avaliadas apresentam dissimilaridade genética quanto às características agronômicas avaliadas, híbridos com maior efeito heterótico podem ser obtidos dos cruzamentos entre Iraí × Goiano Precoce, Iraí × Pérola, Goiano Precoce × CNFC 10467 e IAPAR $81 \times$ Pérola.

A característica comprimento médio longitudinal das vagens é a que mais contribuiu para estimar a diversidade genética entre as cultivares/linhagens e a número médio de sementes por plantas a que menos contribui.

\section{REFERÊNCIAS BIBLIOGRÁFICAS}

ARANTES, E. M.; CREMON, C.; LUIZ, M. A. C. Alterações dos atributos químicos do solo cultivado no sistema orgânico com plantio direto sob diferentes coberturas vegetais. Revista Agrarian, Dourados, v. 5, n. 15, p.47-54, 2012.

BITOCCHI, E.; BELLUCCI, E.; GIARDINI, A.; RAU, D.; RODRIGUEZ, M.; BIAGETTI, E.; SANTILOCCHI, R.; SPAGNOLETTI, Z. P.; GIOIA, T.; LOGOZZO, G.; ATTENE, G.; NANNI, L.; PAPA, R. Molecular analysis of the parallel domestication of the common bean (Phaseolus vulgaris) in Mesoamerica and the Andes. New Phytologist, Chichester, v. 197, n. 1, p.300-313, 2013.

COELHO, C. M. M.; COIMBRA, J. L.; SOUZA, C. A.; BOGO, A.; GUIDOLIN, A. F. Divergência genética em acessos de feijão (Phaseolus vulgaris L.). Ciência Rural, Santa Maria, v. 37, n. 5, p.1241-1247, 2007.

CORREA, A. M.; GONÇALVEZ, M. C. Divergência genética em genótipos de feijão comum cultivados em Mato Grosso do Sul. Revista Ceres, Viçosa, v. 59, n. 2, p.206-212, 2012.

CRUZ, C. D.; CARNEIRO, P. C. S.; REGAZZI, A. J. Modelos biométricos aplicados ao melhoramento genético. v. 2, 3. ed. Viçosa, MG: Editora UFV, 2014. A668 p.

CRUZ, C. D. GENES: a software package for analysis in experimental statistics and quantitative genetics. Acta Scientiarum, Maringá, v. 35, n. 3, p.271-276; 2013.

DALLACORT, R.; NEVES, S. M. A. S.; NUNES, M. C. M. Variabilidade da temperatura e das chuvas de Cáceres/Pantanal Mato-Grossense - Brasil. Geografia, Londrina, v. 23, n. 1. p.21-33, 2014. 
GEPTS, P.; BLISS, F. A. Phaseolin variability among wild and cultivated common bean (Phaseolus vulgaris L.) from Colombia. Economic Botany, New York, v. 40, n. 4, p.469478, 1986.

GONÇALVES, D. L.; BARELLI, M. A, A.; SANTOS, P. R. J..; OLIVEIRA, T, C.; SILVA, C. R. ; NEVES, L. G.; POLETINE, J. P.; LUZ, P. B.. Variabilidade genética de germoplasma tradicional de feijoeiro comum na região de Cáceres-MT. Ciência Rural, Santa Maria, v. 46, n. 1, p.100-107, 2016.

GRIGOLO, S.; FIOREZE, A. C. D. L.; DENARDI, S.; VACARI, J. Implicações da análise univariada e multivariada na dissimilaridade de acessos de feijão comum. Revista de Ciências Agroveterinárias, Lages, v. 17, n. 3, p.351-360, 2018.

MAMBRIN, R. B.; RIBEIRO, N. D.; STORCK, L.; DOMINGUES, L. L.; BARKERT, K. A. Seleção de linhagens de feijão baseada em caracteres morfológicos, fenológicos e de produção. Revista de Agricultura, Piracicaba, v. 90, n. 2, p.141-155, 2015.

MAZIEIRO, S. M.; RIBEIRO, N. D.; CASAGRANDE, C. R. Genetic dissimilarity of common bean lines for agronomic and biofortitication traits. Genetic and Molecular Research, [s. n.], v. 16, n. 3, p.1-13, 2017.

MELO, L. C.; PEREIRA, H. S.; SOUZA, T. L. P. O.; FARIA, L. C.; AGUIAR, M. S.; WENDLAND, A.; CARVALHO, H. W. L.; ALMEIDA, V. M.; MELO, C. L. P.; COSTA, A. F.; ITO, M. A.; PEREIRA FILHO, I. A.; POSSE, S. C. P.; MAGALDI, M. C. S.; CABRERA DIAZ, J. L.; COSTA, J. G. C.; ABREU, A. F. B.; MARTINS, M.; GUIMARÃES, C. M.; TRINDADE, N. L. S. R.; MELO, P. G. S.; BRAZ, A. J. B. P.; SOUZA, N. P.; FARIA, J. C. BRS FC104: cultivar de feijão-comum carioca superprecoce. Santo Antônio de Goiás: Embrapa Arroz e Feijão, 2017. (Comunicado Técnico, 239). Disponível em: https://ainfo.cnptia.embrapa.br/digital/bitstream/item/171825/1/CNPAF-2017-comt239.pdf.

Acesso em: 15 ago. 2018.

OLIVEIRA, A. C.; SILVA, J.; SANTOS, M. M.; CANCELlIER, E. L.; FIDELIES, R, R. Desempenho agronômico de cultivares de feijão em função da adubação fosfatada no sul do Estado do Tocantins. Revista Caatinga, Mossoró, v. 27, n. 1, p.50-59, 2014.

POlETINE, J. P.; SÁBIO, G.; BARElli, M. A. A.; SEVERINO, J. J.; AUGUSTO, J.; SILVA, G. C. M.; BORÁZIO, R. F. Caracterização morfoagronômica e divergência genética de acessos de feijão comum coletados no estado do Mato Grosso e avaliados na região do arenito Caiuá. Journal of Agronomic Sciences, [s. 1.], v. 5, n. 1, p.83-96, 2016.

RIBEIRO, N. D.; SANTOS, G. G.; MAZIEIRO, S.M.; STECKLING, S. M. Phenological, plant architecture, and grain yield traits on common bean line selection. Revista Caatinga, Mossoró, v. 31, n, 3, p.657-666, 2018.

ROTILI, E. A.; CANCELLIER, L. L.; DOTTO, M. A.; PELUZIO, J. M., CARVALHO, E. V. Divergência genética em genótipos de milho, no Estado do Tocantins. Revista Ciência Agronômica, v. 43, n. 3, p.516-521, 2012.

SAlGADO, F. H. M.; FIDELIS, R. R.; CARVALHO, G. L.; SANTOS, G. R.; CANCELlIER, E. L.; SILVA, G. R. Comportamento de genótipos de feijão, no período da 
entressafra, no sul do estado de Tocantins. Bioscience Journal, Uberlândia, v. 27, n. 1, p. 52$58,2011$.

SEBIM, D.E.; OLIVEIRA, P. H.; BRUSAMARELlO, A. P.; BARETTA, D. R.; BRUM, B. Diversidade genética entre populações de feijão crioulo através da análise multivariada de caracteres morfoagronômicos. Espacios, Caracas, v. 37, n. 16, p.19-31, 2016.

SINGH, D. The relative importance of characters affecting genetic divergence. Indian Journal of Genetic and Plant Breeding, New Delhi, v. 41, n. 2, p.237-245, 1981.

SUlZBACHER, L. J.; SILVA, V. P.; ZAGO, B. W.; CORRÊA, C. L.; DUARTE, A. V. M.; BARELLI, M. A. A. Análise da divergência genética através de caracteres agronômicos em genótipos de feijão comum. Espacios, Caracas, v. 38, n. 23, p.26-39, 2017.

TSUTSUMI, C. Y.; BULEGON, L. G.; PIANO, J. T. Melhoramento genético do feijoeiro: avanços, perspectivas e novos estudos, no âmbito nacional. Nativa, Sinop, v. 3, n. 3, p.217$223,2015$.

VALE, N. M.; BARILI, L. D.; OLIVEIRA, H. M.; CARNEIRO, J. E. S.; CARNEIRO, P. C. S.; SILVA, F. L. Escolha de genitores quanto à precocidade e produtividade de feijão tipo carioca. Pesquisa agropecuária Brasileira, Brasília, v. 50, n. 2, p.141-148, 2015.

VIDYAKAR, V.; LAL, G.M.; SINGH, M. K.; KUMAR, A. Study on genetic diversity in French bean (Phaseolus vulgaris L.). Journal of Pharmacognosy and Phytochemistry, New Delhi, v. 6, n. 6, p.184-187, 2017. 